\title{
Impact of a Protocol for the Prevention and Care of Oral Mucositis in Pediatric Patients Diagnosed with Cancer
}

\section{Carlos Ávila-Sánchez*, Jessica Paola Purizaca-Bazán, Georgina Félix-Bermúdez, María Andrea Ellis-Irigoyen, María de Lourdes Vega-Vega and Gabriela Escamilla-Asiaín}

Hospital Infantil Teletón de Oncología, Querétaro, Qro., Mexico

Received for publication: 30 October 2016; accepted for publication: 25 February 2017

Available online: 4 August 2017

\section{KEYWORDS}

Oral mucositis;

Pediatric cancer;

Oral care protocol

\begin{abstract}
Introduction: Oral mucositis $(\mathrm{OM})$ is a common complication associated with cancer treatment, and it can range from mild to severe degree. Up to $80 \%$ of children undergoing chemotherapy will experience some degree of $\mathrm{OM}$, which can get to increase mortality by up to $40 \%$ in severe cases. Although OM incidence differs according to cancer type and treatment regimen, children with hematological malignancies experience higher incidence and preva-i lence than children diagnosed with solid tumors. According to the above, OM is likely to represent a major cause for common protocol-indicated dose reduction and, hence, for treatment delay. Therefore, this work analyzes approaches that been used to impact on OM prevention and opportune treatment, in addition to contributing to yet-unpublished national statistics. Material and Methods: This was a retrospective, descriptive study of 157 cancer-diagnosed pediatric patients attended to during the period from September 2014 to June 2016 and who were at risk for experiencing an $\mathrm{OM}$ event during their treatment stage. Results: $\mathrm{OM}$ occurrence was $21.6 \%$ and annual prevalence was $2.6 \%$ out of a total of 1731 assessments. Conclusions: Our results are promising, and expanding the sample size, as well the perspective of collaboration with other institutions is suggested in order to promote a standardized oral care protocol for children diagnosed with cancer. (creativecommons.org/licenses/by-nc-nd/4.0/).
\end{abstract}

${ }^{*}$ E-mail for correspondence: avila@hospitalteleton.org.mx (C. Ávila-Sánchez) 


\section{INTRODUCTION}

Oncologic problems are characterized by cell proliferation deregulation and increase, as well as apoptosis decrease. Therefore, conventional antineoplastic treatments are targeted to block this proliferation. However, chemotherapy and radiotherapy actions are non-specific, and in the context of antineoplastic treatments it is therefore common to observe harm to tissues that in physiological conditions have high cell-replication rates, since they act on cell multiplicative phase. Among these mechanisms, toxicity to oral and gastrointestinal mucosa stands out, which produces lesions such as mucositis.

$\mathrm{OM}$ is an acute and severe toxic inflammatory reaction that affects the entire gastrointestinal (GI) tract from the oral-labial to the anogenital mucosa, secondary to toxicity of antineoplastic treatments such as radiotherapy, chemotherapy or conditioning for hematopoietic stem cell transplantation (HSCT) used in hematological malignancies. This inflammation can progress until confluent ulcers are formed, thus decreasing patient quality of life owing to pain, malnutrition, delay on treatment administration and increasing the risk of a life-threatening infection and increasing hospital stay and cost of treatment ${ }^{1,2}$.

OM prevalence is $40 \%$ to $100 \%$, depending on the type of cancer and the employed treatment. In adult population, it occurs in approximately in 40 to $76 \%$ of patients on treatment with standard-dose chemotherapy, as well as in 80 to $100 \%$ of patients undergoing HSCT conditioning, and in $100 \%$ of patients undergoing radiotherapy to the head and neck region, especially if the dose exceeds $4.000-6.000 \mathrm{cGy}^{2-5}$.

On the other hand, incidence of $O M$ in children has increased in comparison with adult population; however, there are many variations in the literature, with estimates ranging from 52 to $80 \%{ }^{2}$.

Currently, the literature on $\mathrm{OM}$ in pediatric population is scarce in spite of the fact of the risk to experience this complication being high in comparison with adult population, since there are factors related to patient age that may contribute to its development, such as deficient oral hygiene practices and inadequate nutritional status ${ }^{2,4-6}$.

Patients with $O M$ usually experience a dry mouth sensation, difficulty to swallow, burning, tingling in the lips, pain, etc. One of the most serious complications is the development of infections (especially with herpes simplex virus or Candida albicans), with an increased risk in patients with prolonged neutropenia and which may threaten the patient's life ${ }^{5,7}$. Currently, there is no appropriate clinical prophylaxis or efficacious antidote for $\mathrm{OM}$, and management is therefore mainly focused on symptom palliation and infection prevention.

There are different methods to assess and quantify the changes produced in the oral epithelium as a side effect of antineoplastic treatment. Among scale-based classifications, "general" mucositis assessment scales and "multiple-variable" OM assessment scales can normally be differentiated. The former are usually comprised by 4 or 5 variables and allow for overall status of the mouth to be associated with mucositis. In contrast, the second type of scales assesses different variables and their relationship with oral health status and function; the obtained scores are added, and a mucositis severity overall assessment is obtained ${ }^{1-6}$.
In 1979, the World Health Organization (WHO) defined MO lesions status according to their severity by assessing tolerance to the oral route for food ingestion and being able to speak, with five grades, from 0 to IV, being established in patients. Meanwhile, the National Cancer Institute presented in 1998 an update of the Common Toxicity Criteria (CTC), where $\mathrm{OM}$ caused by radiation, chemotherapy and OM deriying from HSCT are differentiated, with OM being classified according to the zone of appearance (assessed areas: right and left jugal mucosa, hard and soft palate, lingual dorsum, lateral borders of the tongue, floor of the mouth). Of both classifications, the one that includes tolerance to the orat route, pain, oral cavity clinical characteristics and patient speech capability is the WHO scale and, therefore, it is the one that was chosen to be used in our hospital center for $\mathrm{OM}^{\circ}$ classification.

In addition to taking the $\mathrm{MO}$ classification into account, it is important for predisposing factors to be identified, includ ing: a neglected oral cavity, dental pathology and high doses of certain antineoplastic agents (chemotherapy and radiotherapy). Oral cavity health status prior to the disease is one of the main conditioning factors for the development of mucositis, its degree, duration and evolution. Other factors that modulate $\mathrm{OM}$ symptoms are blood diseases with oraf manifestations, cytostatic agents and radiotherapy, as well as immunodepression state or coexistence of other systemic diseases such as diabetes, renal failure or organ transplant. Mucositis severity can vary according to the type, dose, frequency and chronicity of the antineoplastic treatment received by the patient, although there are treatments that are particularly related to its occurrence. However, chemo therapy and radiotherapy actions are not specific, and in the context of antineoplastic treatments it is therefore common to observe damage to tissues that in physiologic conditions have high cell replication rates. Among them, oral and GI mucosa toxicity stands out ${ }^{1-6}$.

According to a correct classification and identification of risks, treatment modalities can be non-pharmacological or with pharmacological agents such as lidocaine and morphine, which are used for the management of pain and as palliatives. Non-pharmacological treatments include measures of oral hygiene, mild mouthwashes, cryotherapy, etc. Recently, photobiomodulation (laser therapy) has been used in different aspects of dentistry, since it is a safe non-phar macological method that can modulate several metabolic processes by means of energy absorption by macrophag $\mathrm{es}^{3-5,7-10}$.

Low-level laser therapy (LLLT) acts on mitochondriat respiration and leads to an increase in the production of Adenosine Triphosphate (ATP), which produces intracellular, reactive oxygen species. These changes result in fibroblast proliferation, collagen synthesis, inflammatory response adjustment, and they also induce angiogenesis and tissue repair. There are different explanations for the reduction in pain caused by photobiomodulation, for example, inflammatory process modulation, excitation alteration, peripherat nerve nervous conduction and endogenous endorphin release stimulation. Therefore, LLLT is considered for preventive and palliative treatment of $\mathrm{OM}^{1,4,5}$. LLLT with laser diodes of several wavelengths (630-680, 700-830 and $900 \mathrm{~nm}$ ) has been reported to be an efficacious, simple and non-invasive technique in the treatment of $\mathrm{OM}$, without toxicity 
Table 1. Protocol for oral mucositis prevention

\begin{tabular}{l} 
Intervention \\
Therapeutics A \\
Dental examination \\
Oral hygiene \\
Tooth brushing \\
Mild mouthwashes \\
Antiseptics \\
Lip moisturizing \\
Therapeutics B \\
Low-level laser therapy (LLLT) \\
- Beam: infrared. \\
- Output power: 300 mW. \\
- Wavelength: 980 nm. \\
- Dose/Energy density: 18 J/cm ${ }^{2}$. \\
- Frequency: 8000 Hz. \\
- Application: single point instead of scanning in \\
motion for 8 continuous minutes or fractioned in two \\
4-minute episodes, according to patient cooperation. \\
- Duration: \\
a) Start 24 hours after high-dose methotrexate \\
administration or at the beginning of radiotherapy \\
at the head and neck level or at the start of \\
HSCT conditioning thrice weekly. \\
b) Apply at the completion of chemotherapy with \\
standard-dose methotrexate in a single session. \\
\hline
\end{tabular}

being observed in clinical trials and, and lesions within the oral cavity have therefore been suggested to likely respond better to LLLT than skin wounds, since the oral region has higher blood irrigation ${ }^{4-6}$.

Having interdisciplinary support in hospital centers is of vital interest. Hence, we consider important assessing the OM care protocol in pediatric cancer patients with the purpose to improve quality of life and translate this into adequate clinical evolution.

\section{GENERAL PURPOSE}

To analyze the impact of implementing a standardized protocol of hospital oral care and the record of OM incidence in immunocompromised pediatric cancer patients undergoing treatment with chemotherapy, radiotherapy and/or hematopoietic stem cell transplantation (HSCT).

\section{Hypothesis}

Our dental care protocol in patients starting antineoplastic treatment reduces the prevalence of OM in comparison with the prevalence reported in the international literature.

\section{MATERIAL AND METHODS}

\section{Study group}

This was a cross-sectional, retrospective study. The sample consisted of records of pediatric population younger than 18 years diagnosed with cancer starting treatment in the outor inpatient setting during the period from September 2014 to June 2016 and who were referred to the pediatric dentistry department for the first time and subsequently. After each primary assessment, and according to the specialist's criterion, a preventive or therapeutic dental protocol with general measures and/or laser therapy was started (Table 1). For patients with oncologic assessment who started a protocol with high-dose chemotherapy with methotrexate of who underwent head and neck radiotherapy, the therapeutic protocol implemented in our center by the department of pediatric dentistry was directly initiated. Data were collected in an electronic database on order to analyze treat ed patients' epidemiological characteristics, as well as OM prevalence and to assess the implemented dental protocols.

\section{Inclusion criteria}

Patients of the female or male gender, younger than 18 years of age, diagnosed with pediatric cancer, who received dental care in our hospital center.

\section{Exclusion criteria}

Patients of the female or male gender, younger than 18 years, not diagnosed with pediatric cancer, who received care in our hospital center.

\section{Elimination criteria}

Patients counter-referred to other hospital unit, healthy pa tients who were candidates to bone marrow donation.

\section{General description of the study}

This was a retrospective, descriptive study that included electronic medical file and registry log data from 157 patients diagnosed with pediatric cancer attended to during the pe riod from September 2014 to June 2016. Data were obtained from registry logs of the hospital's Departments of Pediatric Dentistry, Infectology, Clinical Laboratory and Pathology. Of the 157 patients attended to, 1731 records related to firsttime and subsequent dental care were obtained, which were recorded in electronic spreadsheets and, subsequently, the OM relationship was analyzed by specific oncologic pathology (Grades I, II, III or IV according to WHO criteria). Subsequently, data were analyzed according to the occurrence of $\mathrm{OM}$ in the groups of higher frequency, and the preventive measures and/or dental treatment used were retrospectively assessed. Data were analyzed in the SPSS V21 Program, and the graphs in the GraphPad Prism 5 program.

\section{RESULTS}

\section{Epidemiological characteristics of the study population}

Of a total of 157 patients, a mean age of $7.9(S D \pm 5.1)$ years was recorded, with $20 \%$ being females and $80 \%$ males. Patients were classified in three groups according to the associated pediatric oncologic pathology based on the Seguro 
Table 2. Epidemiological characteristics

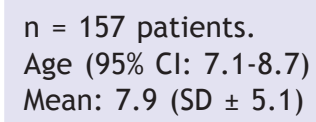

ALL: Acute Lymphoblastic Leukemia; AML: Acute Myeloid Leukemia; BL: Burkitt Lymphoma; CGL: Chronic Granulocytic Leukemia; CNS: Central Nervous System; HCL: Langerhans Cell Histiocytosis; HL: Hodgkin's Lymphoma; NHL: Non-Hodgkin's Lymphoma.

Popular (people's health insurance) National Program reference. In order of frequency, hematological malignancies were the most frequent with $43 \%$, followed by solid tumors in $39 \%$ and central nervous system (CNS) tumors in $18 \%$. Further description is made in table 2.

Of the totality of patients with cancer diagnosis, firsttime assessment by the pediatric dentistry department is described. Nearly half the patients (48\%) had caries and non-complete oral cavity integrity (48\%). Dental evaluations are detailed in table 3 .

\section{Frequency of oral mucositis by oncologic condition}

Of the 157 patients attended to, 34 had at least one mucositis event, i.e., an occurrence rate of $21.6 \%$ over the study period. Annual prevalence of at least one OM event in one year within the analyzed period was $2.6 \%$ out of 1731 total assessments by the pediatric dentistry department. OM grades were assessed according to the WHO classification (Table 4). Ninety-four percent of recorded OMs corresponded to Grade I and Grade II, and only 4\% to Grade III and 2\% to Grade IV. OM and oncologic pathology diagnosis were associated according to the percentage of occurrence, with a
Table 3. Oral health in pediatric patients diagnosed with cancer

Dental diagnosis according to first-time assessment by the Pediatric Dentistry Department

$\begin{array}{ll}\begin{array}{l}\text { Oral cavity integrity } \\ \text { (soft tissues) }\end{array} & 48 \% \\ \text { Caries } & 48 \% \\ \text { Other } & 4 \%\end{array}$

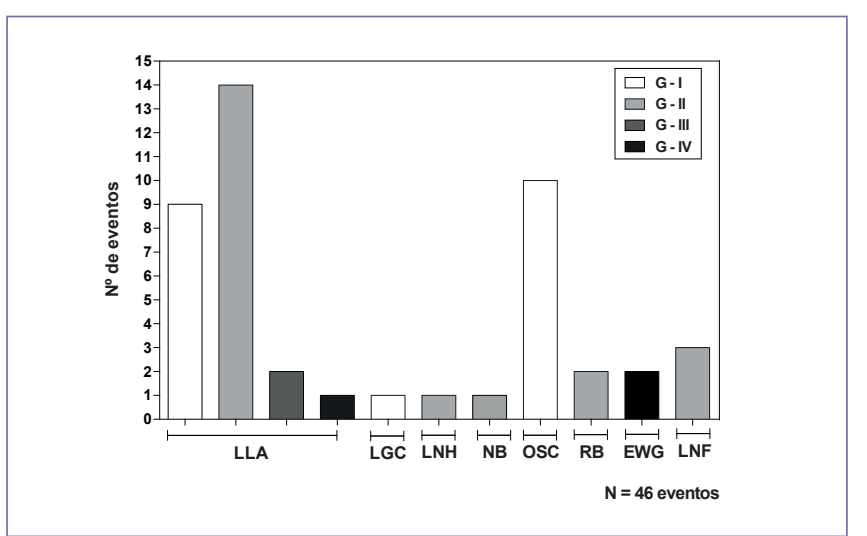

Figure 1. Oral mucositis grades associated with oncologic pathologies. The bars represent main associated pathologies occurrence by mucositis severity grade. Bars are shown in shades of gray according to severity grade. ALL: Acute Lymphoblastic Leukemia; CGL: Chronic Granulocytic Leukemia; EWG: Ewing Sarcoma; LBL: Lymphoblastic Lymphoma; NB: Neuroblastoma; NHL: Non-Hodgkin Lymphoma; OSC: Osteosarcoma; $\mathrm{RB}$ : Retinoblastoma.

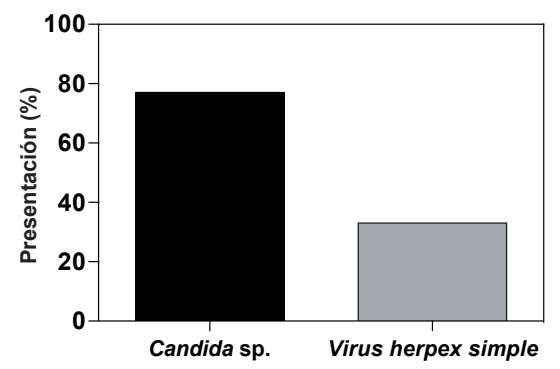

Figure 2. Oral mucositis-associated microorganisms. The black bar shows the percentage of Candida sp occurrence; Herpes simplex occurrence is shown in gray.

higher number of recorded events corresponding to children with diagnoses of acute lymphoblastic leukemia and osteosarcoma (Fig. 1).

\section{Microorganisms associated with oral mucositis events}

Of the recorded OM events, samples were taken for basic morphological staining with the purpose to identify yeasts 
Table 4. Antineoplastic therapy used and associated mucositis grades

\begin{tabular}{lcll}
\hline $\begin{array}{l}\text { Acute Lymphoblastic } \\
\text { Leukemia** }\end{array}$ & & Osteosarcoma* \\
\hline Grade I Mucositis & $\%$ & Grade I Mucositis & $\%$ \\
Very High Risk & 17 & MAPIE & 36 \\
Standard & 33 & MAP & 64 \\
High Risk & 50 & & \\
Grade II Mucositis & $\%$ & & \\
Standard & 46 & \\
High Risk & 33 & \\
Very High Risk & 21 & \\
Grade III Mucositis & $\%$ & \\
Standard & 50 & \\
High Risk & 50 & \\
Grade IV Mucositis & $\%$ & \\
High Risk & 100 & \\
\hline
\end{tabular}

*MAP: Methotrexate, Doxorubicin, Cisplatin.

MAPIE: Methotrexate, Doxorubicin, Cisplatin, Etoposide, Ifosfamide. **For the leukemia protocol, patients receive antineoplastic therapy according to their treatment stage with: Methotrexate, AraC,

Vincristine, Dexamethasone, etc.

Table 5. Association and risk for experiencing oral mucositis and dental therapeutics used

\begin{tabular}{lcc}
\hline Pathology & ${ }^{*} \mathrm{p} \leq 0.05$ & $\mathrm{RR}$ \\
\hline Leukemias & No & 0.6 \\
Osteosarcomas & No & 0.32 \\
\hline
\end{tabular}

*Fisher's exact test.

(KOH staining) or intracytoplasmic inclusions (Tzank), which help to infer the type of infection with Candida sp or with herpes simplex, respectively. Seventy-seven corresponded to morphological findings positive for related yeasts, of patients mostly diagnosed with leukemia, and 33\% related to intracytoplasmic inclusions (Fig. 2).

\section{Association of dental treatment used and risk for $O M$}

Fischer's exact test was used to analyze the relationship between OM grades in patients diagnosed with leukemia and osteosarcoma and the therapeutics used according to dental assessment (Table 5).

\section{Description of therapeutic protocols used by the department of pediatric dentistry}

Patients with first-time or subsequent dental evaluation, as well as patients at risk for the development of OM (leukemia and osteosarcoma), received general measures and/ or low-level laser therapy. According to the diagnosed OM grade, the therapeutic protocol described in Table 6 was implemented.
Table 6. Oral mucositis therapeutic protocol

WHO Grade I oral mucositis

Tooth brushing

Mild mouthwashes

Antiseptics

Lip moisturizing

Low-level laser therapy

- Beam: infrared

- Output power: $300 \mathrm{~mW}$

- Wavelength: $980 \mathrm{~nm}$

- Dose/Energy density: $18 \mathrm{~J} / \mathrm{cm}^{2}$

- Frequency: $8000 \mathrm{~Hz}$

Nutrition

WHO Grade II oral mucositis

Tooth brushing

Mild mouthwashes

Antiseptics

Lip moisturizing

Low-level laser therapy

- Beam: infrared

- Output power: $300 \mathrm{~mW}$

- Wavelength: $980 \mathrm{~nm}$

- Dose/Energy density: $18 \mathrm{~J} / \mathrm{cm}^{2}$

- Frequency: $8000 \mathrm{~Hz}$

- Application: single point instead of scanning in motion for 8 continuous minutes or fractioned in two 4-minute episodes, according to patient cooperation

- Session: daily

- LLLT use contraindicated in zone with diagnosed or suspected infection

Anti-inflammatory / Analgesic / Topical anesthetic

Mucoprotector

- Gel with polyvinylpyrrolidone, benzalkonium chloride, sodium hyaluronate and sodium benzoate

Nutrition

Pain medicine

Infectology / Pathology

- Clindamycin $40 \mathrm{mg} / \mathrm{kg} /$ day for infection risk

WHO Grade III and IV oral mucositis

Tooth brushing

Mild mouthwashes

Antiseptics

Lip moisturizing

Low-level laser therapy

- Beam: infrared

- Output power: $300 \mathrm{~mW}$

- Wavelength: $980 \mathrm{~nm}$

- Dose/Energy density: $18 \mathrm{~J} / \mathrm{cm}^{2}$

- Frequency: $9000 \mathrm{~Hz}$

Anti-inflammatory / Analgesic / Topical anesthetic - Benzydamine 5 to $10 \mathrm{ml}$ solution

Mucoprotector

Artificial saliva

Nutrition

Pain medicine / Palliative care

Infectology / Pathology 


\section{DISCUSSION}

Oral health remains a public health problem in Mexico. Our data are consistent with what the Ministry of Health's last report reflects ${ }^{11}$, where $50 \%$ of children have some dental problem, especially caries. In addition to oral health status, cancer-diagnosed pediatric patients have to face a new organic challenge. For this reason, oral health should be linked within the oncologic support our patients receive.

As in many international reference centers ${ }^{12}$, implementation of both OM preventive and therapeutic protocols is being initiated; however, there are no definitive national guidelines for its treatment in pediatric population. Children have been shown to be at higher risk for developing OM than adults ${ }^{13}$, and poor control of this condition can have a negative outcome in children's development.

With the protocol implemented in our center, a prevalence of about $22 \%$ is observed, which is less than half the prevalence reported in global literature ${ }^{2,14}$. Furthermore, more than $90 \%$ of oral mucositis cases had grade I and grade II presentations and were resolved according to MO therapeutic management protocols in less than 7 days.

An intact oral mucosa creates a physical barrier against pathogens and provides clearance of mucosacolonizing microorganisms thanks to the detachment of epithelial cells. Once the mucosal barrier is ruptured, infection occurs, mainly with colonizing microorganisms such as Candida species, mainly albicans, Streptococcus of the viridans group and herpes simplex virus (HSV) infection reactivation. Our data on $\mathrm{OM}$-associated microorganisms' frequency are consistent with those reported in the literature, where most oral infections in patients are produced by Candida albicans, with the incidence in series of patients with leukemia who received chemotherapy being from $21.8 \%$ to $58 \%$, and in series of patients with solid tumors, up to $70 \%$. With regard to HSV infection, it occurs in $21 \%$ to $90 \%$ of seropositive patients who receive high-dose chemotherapy, especially in transplanted patients. This infection is associated with severe grades of mucositis ${ }^{15-17}$.

According to our observations, implementation of good oral hygiene and the use of a standardized oral care protocol for all OM-susceptible children is as important as the use of therapeutic laser, since the non-significant association between using or not low-level laser does not decrease the risk to suffer OM. In the international literature there is an important number of preventive oral health care protocols ${ }^{18-20}$; however, national reference reports are not yet available. Evidence on efficacious pharmacological treatments in the treatment of oral mucositis is insufficient, and it is limited to clinical trials in adults with drugs such as recombinant keratinocyte growth factor. A multimodal approach to oral cavity adequate care with general measures such as those proposed in our work (Therapeutics A and B), and interdisciplinary management between the departments of infectology, pain medicine, psycho-oncology and dentistry, may help to decrease oral mucositis duration and severity during antineoplastic treatment in pediatric patients by giving support and reducing delays in the management provided by the pediatric oncologist.

\section{CONCLUSIONS}

Oral care protocols can offer OM reduction in children undergoing antineoplastic therapy. Our results are promising, although interpreting them with a larger number of patients is necessary, as well as research in multi-institutional collaboration in order to define and promote the optimal oral care regimen in children diagnosed with pediatric cancer.

\section{ACKNOWLEDGEMENTS}

We would like to thank Dr. Isaac Urrutia Ballesteros, of the Department of Pain Medicine, for his contribution to analgesic management support within the mucositis care therapeu? tic protocols. We also express our gratitude to MS Gina de Gasperín Estrada for her collaboration in data acquisition.

\section{CONFLICT OF INTERESTS}

The authors declare no conflicts of interests.

\section{REFERENCES}

1. Multinational Association of Supportive Care In Cancer and International Society of Oral Oncology. MASCC/ISOO. Evidence-based Clinicat Practice Guidelines for oral mucositis secondary to cancer therapy. MASCC/ISOO MO Study Group. 2014; 1-8.

2. Qutob A, Allen G, Gue S, Revesz T, Logan R, Keefe D. Implementation of a hospital oral care protocol and recording of oral mucositis in children receiving cancer treatment. A retrospective and prospectiye study. Support Care Cancer. 2013; 21:113-1120.

3. Mallick S1, Benson R2, Rath GK2. Radiation induced oral mucositis: a review of current literature on prevention and management. Eur Arch Otorhinolaryngol. 2016 Sep;273(9):2285-93.

4. Fekrazad R, Chiniforush N. Oral mucositis prevention and management by therapeutic laser in head and neck cancers. J Lasers Med Sci. 2014 Winter; 5(1): 1-7.

5. Lalla VR, Bowen J, Barasch A, Elting L, Epstein J, Keefe MD, et al: MASCC/ISOO Clinical Practice Guidelines for the Management of orat mucositis secondary to cancer therapy. Cancer. 2014; 1453-1461.

6. Maguire BD, Fulton SJ, Park J, Brown GC, Correa PME, Eilers J, et al. Systematic review of basic oral care for the management of oral mucositis in cancer patients. Support Care Cancer. 2013; 21: 3165-3177.

7. Saunders PD, Epstein BJ, Elad S, Allemano J, Bossi P, van de Wetering $\mathrm{DM}$, et al. Systematic review of antimicrobials, mucosal coating agents, anesthetics, and analgesics for the management of oral mucositis in cancer patients. Support Care Cancer. 2013; 21:3191-3207.

8. Nicolatou-Galitis O, Sarri T, Bowen J, Di Palma M, Kouloulias EV, Nis cola $\mathrm{P}$, et al. Systematic review of anti-inflammatory agents for the management of oral mucositis in cancer patients. Support Care Cancer. 2013; 21:3179-3189.

9. Yarom N, Ariyawardana A, Hovan A, Barasch A, Jarvis V, Jensen BS, eto al. Systematic review of natural agents for the management of orat mucositis in cancer patients. Support Care Cancer. 2013; 21:3209-3221.

10. Jensen BS, Jarvis V, Zadik Y, Barasch A, Ariyawardana A, Hovan A, et al. Systematic review of miscellaneous agents for the management of oral mucositis in cancer patients. Support Care Cancer. 2013; 21:3223-3232.

11. Archivo Online. Perfil epidemiológico de la salud bucal en México 2010. Secretaría de Salud - Subsecretaría de prevención y promoción de la salud dirección general de Epidemiología. http://www.epidemiologia. salud.gob. $\mathrm{mx} /$

12. Sung L, Robinson P, Treister N, Baggott T, Gibson P, Tissing W, et al. Guideline for the prevention of oral and oropharyngeal mucositis in children receiving treatment for cancer or undergoing haematopoietic stem cell transplantation. BMJ Supportive \& Palliative Care 2015; 1-10. 
13. Cheng KK, Chang AM. Palliation of oral mucositis symptoms in pediatric patients treated with cancer chemotherapy. Cancer Nurs. 2003; 26(6):476-484

14. Otmani N, Alami R, Hessissen L, Mokhtari A, Soulaymani A, Khattab. Determinants of severe oral mucositis in paediatric cancer patients: a prospective study. Int J Paediatr Dent. 2011; 21(3):210-216.

15. Worthington, Clarkson. Prevention of oral mucositis and oral Candidiasis for patients with Cancer treated with chemotherapy: Cochrane Systematic Review. Journal of Dental Education. 2002.66(8):903-11.

16. Chen, Hou, Chow, Chen YC, Hsueh PR, Tien HF. The impact of oral herpes simplex virus infection and candidiasis on chemotherapy-induced oral mucositis patients with hematological malignancies. Eur J Clin Microbiol Infect Dis.2011;30:753-59.
17. Jain, Shah, Chandolia, et al. The Oral Carriage of Candida in Oral Cancer Patients of Indian Origin Undergoing Radiotherapy and/or Chemotherapy. Journal of Clinical and Diagnostic Research. 2016;10(2).

18. Rubio IT1, Cao Y, Hutchins LF, Westbrook KC, Klimberg VS. Effect of glutamine on methotrexate efficacy and toxicity. Ann Surg. 1998; 227(5):772-780.

19. Miller M, Donald D, M. Hagemann T. Prevention and Treatment of Oral Mucositis in Children with Cancer. J Pediatr Pharmacol Ther. 2012; $17(4): 340-50$.

20. Cheng KKF, Chang AM, Yuen MP. Prevention of oral mucositis in paedi atric patients treated with chemotherapy; a randomised crossover trial comparing two protocols of oral care. European Journal of Cancer 2004; 40(8):1208-1216. 